\title{
Low dimensionality semiconductors : modelling of excitons via a fractional-dimensional space
}

\author{
P. Christol, P. Lefebvre and H. Mathieu \\ Groupe d'Etudes des Semiconducteurs, Université Montpellier II, C.N.R.S., PI. E. Bataillon, \\ Case 074, 34095 Montpellier Cedex 5, France
}

(Received 10 November 1992, accepted 9 February 1993)

\begin{abstract}
Résumé. - Nous utilisons un espace des interactions doté d'une dimension fractionnaire pour calculer simplement l'énergie de liaison des excitons confinés dans les puits quantiques, superréseaux et fils quantiques. Une formulation très simple donne cette énergie en fonction de la dimensionalité non-entière de l'environnement physique de la paire électron-trou. Le problème revient alors à déterminer cette dimensionalité $\alpha$, dont nous montrons qu une expression peut être déduite des caractéristiques de la microstructure. $\alpha$ varie continûment de 3 (matériau massif) à 2 pour un puits quantique ou un superréseau, et de 3 à 1 pour un fil quantique, selon le confinement du mouvement des porteurs. Les comparaisons avec d'autres calculs théoriques et données expérimentales sont toujours très convenables, et cette théorie décrit d'une façon cohérente les limites tridimensionnelles du puits quantique $\left(L_{\mathrm{w}} \rightarrow 0\right.$ et $\left.L_{\mathrm{w}} \rightarrow \infty\right)$ et toute la gamme des périodes du superréseau. Un tel modèle, qui ne vise pas à concurrencer les calculs variationnels très précis mais souvent complexes, présente, de par sa souplesse, un grand intérêt pour les spectroscopistes.

Abstract. - An interaction space with a fractionnal dimension is used to calculate in a simple way the binding energies of excitons confined in quantum wells, superlattices and quantum well wires. A very simple formulation provides this energy versus the non-integer dimensionality of the physical environment of the electron-hole pair. The problem then comes to determining the dimensionality $\alpha$. We show that the latter can be expressed from the characteristics of the microstructure. $\alpha$ continuously varies from 3 (bulk material) to 2 for quantum wells and superlattices, and from 3 to 1 for quantum well wires. Quite a fair agreement is obtained with other theoretical calculations and experimental data, and this model coherently describes both threedimensional limiting cases for quantum wells $\left(L_{\mathrm{u}} \rightarrow 0\right.$ and $\left.L_{\mathrm{u}} \rightarrow \infty\right)$ and the whole range of periodsof the superlattice. Such a simple model presents a great interest for spectroscopists though it does not aim to compete with accurate but often tedious variational calculations.
\end{abstract}

\section{Introduction.}

Optical properties of quantum wells (QW's), superlattices (SL's) or quantum-well wires (QWW's) generally involve quantum-confined excitons, - on which a great deal of interest was laid, during the past few years. More particularly, many theoretical works dealt with the 
calculation of excitonic binding energies and oscillator strengths in such systems, using variational approaches of increasing accuracy and complexity $[1,16]$. Depending on the authors, different shapes were chosen for the trial wave functions, and the energy-minimisation procedures were either purely numerical $[1-13,15,16]$ or analytical [14], in some favourable but limited cases. As a simplification, Leavitt and Little [17] and Campi and Villavecchia [18] recently proposed useful scaling laws which are best-fittings to the results of variational calculations. In this paper, we present a completely different approach of excitons in systems with reduced dimensionality. This approach is similar to the ones previously developed in the case of strongly anisotropic media [19-23], such as lamellar or chainlike semiconductors. Our model is based on the fact that, e.g. in a QW, the relative motion of bound electron-hole pairs is neither purely tridimensional (3D) nor bidimensional (2D), but rather $\alpha$-dimensional ( $2<\alpha<3$ ) [23-26]. This motion can thus be considered as isotropic in an $\alpha \mathrm{D}$ space rather than anisotropic in a 3D space. Although the mathematical treatment of this situation is quite tedious, it leads to a very convenient formulation of the exciton wavefunctions, oscillator strengths and energy levels $[19,23]$. The latter can be expressed :

$$
E_{n}=\frac{E_{0}}{\left[n+\frac{\alpha-3}{2}\right]^{2}}
$$

where $E_{0}$ is the $3 \mathrm{D}$ effective Rydberg. Setting $\alpha=3,2$ or 1 allows to obtain the well-known results for the integer-dimensional cases. Of course, the present formalism is only relevant when an anisotropic mediurn surrounds the exciton, and not when a perturbating potential breaks its internal structure, as could occur in type II systems, where electrons and holes are confined in two different materials. Following this formalism, the value of the binding energy of excitons can be easily extracted from the direct measurement of the splitting between $1 \mathrm{~s}$ and 2 s levels [25]. We shall show below that an accurate description of confined excitons is obtained by using a model of the "compression " undergone by the unit vector along the quantization axis of the QW or SL, or by the two unit vectors in the plane perpendicular to the axis of a linear QWW.

\section{Quantum wells.}

In quantum wells, the projection of the relative motion of the bound electron-hole pair along the growth axis is spatially reduced, if compared to the isotropic 3D case. From the consideration of the respective extentions of the electron-hole pair in a $Q W$ and in a 3D exciton, we can infer an expression for the anisotropy parameter $\alpha$. Details on the way to obtain a proper variation law have been given elsewhere [25]. A valuable one is the following:

$$
\alpha=3-\mathrm{e}^{-L_{w}^{*} / 2 a_{0}}
$$

where $a_{0}$ is the effective $3 \mathrm{D}$ Bohr radius and $L_{\mathrm{w}}^{*}=L_{\mathrm{w}}+2 / k_{\mathrm{b}}$ the "effective width " of the quantum well, i.e. the real width $L_{\mathrm{w}}$ plus the spreading of the carriers into the barriers at both sides of the well. By a simple envelope function calculation of the electron and hole confined states in the quantum well, we can estimate this spreading as twice the quantity $k_{\mathrm{b}}^{-1}=k_{\mathrm{be}}^{-1}+k_{\mathrm{bh}}^{-1}, k_{\mathrm{be}}$ and $k_{\mathrm{bh}}$ being the «wave vectors » characteristic of the vanishing of electrons and holes respectively in the barriers. Of course, this simple description is not accurate enough to account for some extreme situations, such as the so-called marginallyconfined excitons, where one type of carriers is confined within a very shallow potential well. In such a case, a more microscopic insight into the electron-hole relative motion is necessary to describe its real spatial extension. Figure 1 shows the good agreement obtained between this 


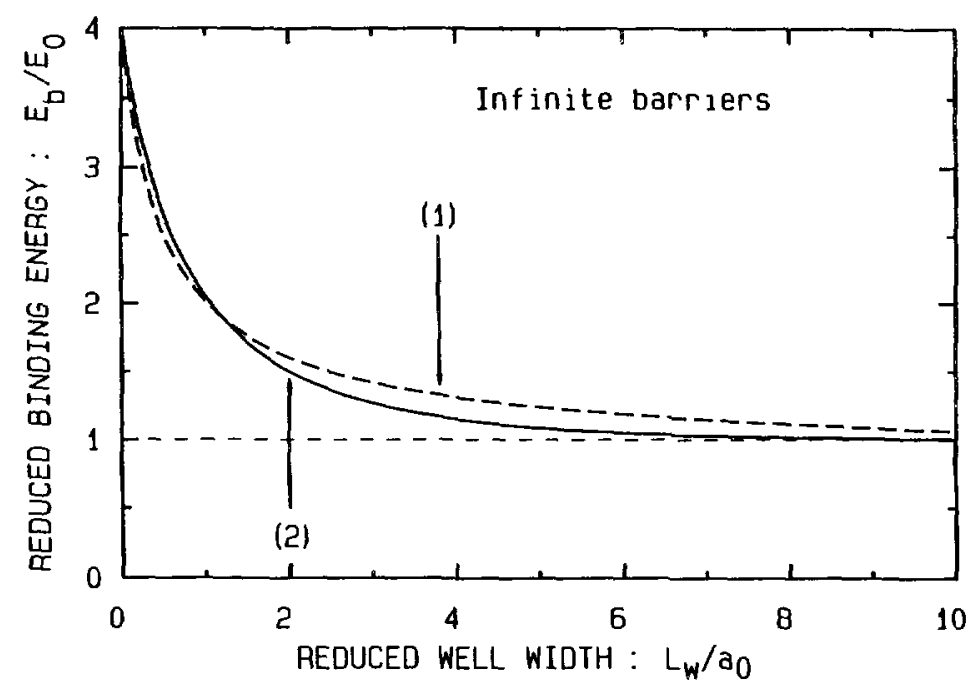

Fig. 1. - Dimensionless exciton binding energy versus the dimensionless well width, for an infinitely deep quantum well. The dashed curve (1) was taken from Bastard et al. [1], while the solid curve (2) was obtained by using equations (1) and (2).

model and that of Bastard [1], for infinitely deep quantum wells. For finite QW's, we obtain the convergence towards a unique $3 \mathrm{D}$ value for light- and heavy-hole exciton binding energies on both limits of infinitely wide and vanishingly narrow quantum wells. The accuracy can even be improved by inclusion of additional ingredients [25] (band nonparabolicities, dielectric constant mismatch between host materials..). In any case, our results compare very favourably to the ones of other calculations and to experimental findings, as shown in figures 2 and 3.

\section{Superlattices.}

A superlattice can be considered as an effective semiconductor with a strongly anisotropic Brillouin zone, which one has the opportunity to vary at will, since the width of this zone along the growth axis is inversely proportional to the period. When the width of the Brillouin zone is large, comparing with $1 / a_{0}$, the Coulombic potential is slowly varying relatively to the period of the superlattice. This corresponds to the criterion for validity of the Wannier approximation. On the other hand, when the wells are decoupled, this criterion is no longer fulfilled, and another type of treatment is necessary. In such a « Wannier-like » regime, the dimensionality $\alpha$ can be expressed :

$$
\alpha=3-(1-\beta)
$$

where $\beta$ is a mass-anisotropy parameter defined as $\beta=\mu_{0 z} / \mu_{z} \cdot \mu_{0 z}$ and $\mu_{z}$ are the on-axis reduced effective masses in the $3 \mathrm{D}$ crystal and in the superlattice, respectively. The latter is obtained by calculating the curvatures of the electron and hole first minibands at the zone center. $\alpha$ varies from 3 for periods near zero. $(\beta \rightarrow 1)$, to 2 when the period (or simply the barrier) becomes larger, i.e. when $\mu_{\text {z }}$ becomes infinite $(\beta \rightarrow 0)$. This means that the dispersion relations of the superlattice run from a $3 D$ to a $2 D$ situation. From equation ( 1 ), the binding energy should consequently vary from one time to four times the effective 3D Rydberg. Of course, this latter value is not reasonable, since equation (3). only traces the inter-well 


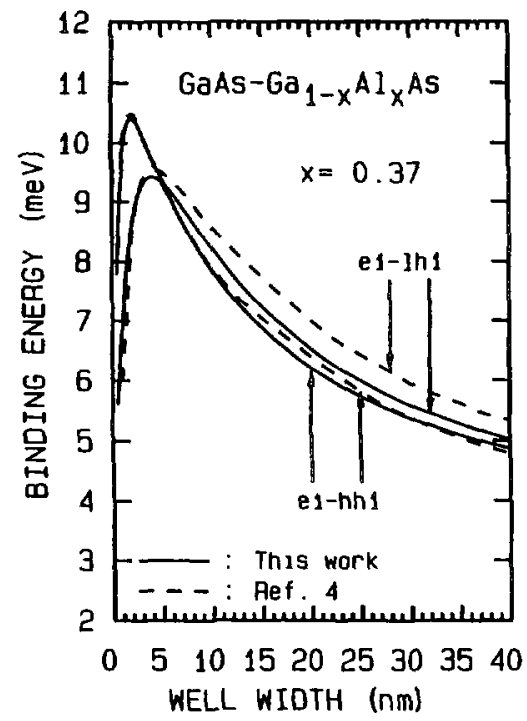

Fig. 2. - Binding energies of the first light-hole (el-lhl) and first heavy-hole (el-hhl) excitons in a $\mathrm{GaAs}_{-} \mathrm{Ga}_{1,}, \mathrm{Al}_{1} \mathrm{As}$ quantum well $(x=0.37)$, versus the well width. Dashed curves were obtained by Priester et al. [4], while solid curves result from equations (1) and (2), using exactly the same hypotheses and numerical parameters. Note that the relative energy position of el-lhl and el-hhl are the same in both models. Moreover, the maximum values and the corresponding well widths are also the same.

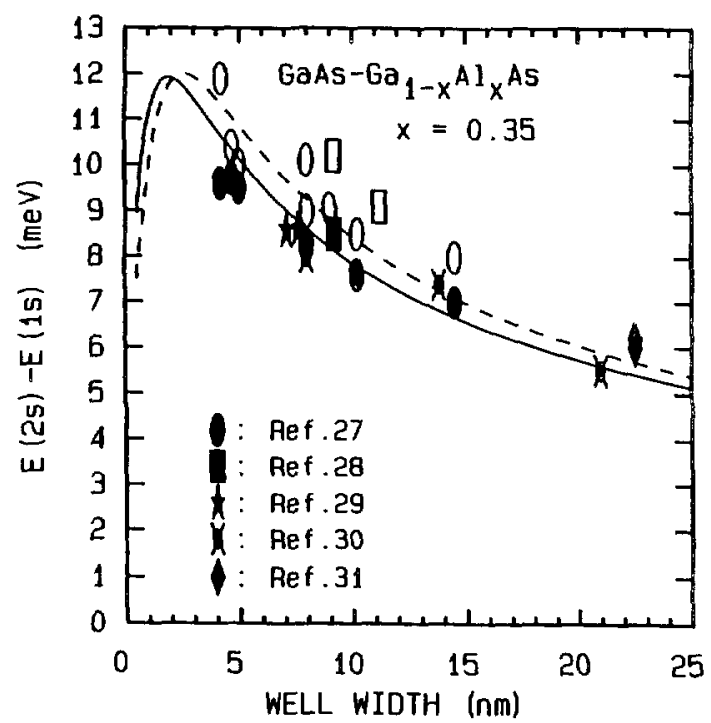

Fig. 3. - Comparison of calculated and measured energy differences $E_{2}, E_{1}$, for GaAs-Ga, $A a_{1} A s$ quantum wells. The calculation was performed with $x=0.35$ and many physical ingredients were included (conduction band nonparabolicity, effective-mass and dielectric constant mismatch). The basic parameters are the same as in reference [16]. Heavy-hole excitons are represented by full figures and solid curve, while open figures and dashed curve were used for light-hole excitons. 
coupling, which is only partly responsible for the anisotropy of the Coulombic interaction. In other words, when the quantum wells become decoupled, we are far away from « Wannierlike » conditions. In fact, for large periods or wide barriers, the bound electron-hole pair is now contained, as a whole, within a given well, i.e. within one elementary cell of the SL. In such a «Frenkel-like » regime, a formulation of $\alpha$ closer to the one of equation (2) should be more appropriate. In a realistic description, both inter-well and intra-well effects should be mixed, as in the following expression :

$$
\alpha=3-(1-\beta) \cdot \mathrm{e}^{-L_{w}^{*} / 2 a_{0}}
$$

Figure 4 shows the Rydberg energies obtained by using equation (4) and equation ( 1 ), for the case of symmetrical $\mathrm{GaAs}-\mathrm{Ga}_{1}, \mathrm{Al}_{1}$ As superlattices, versus the period. These results are compared to the ones of variational calculations and to available experimental data. The overall agreement of the latter with our calculation is very good. For wide periods, where the intra-well effect is dominant ( Frenkel-like » excitons), our result nicely fits the one of Dignam and Sipe [13]. Both models also correctly describe the threshold of appearance of the inter-well coupling (around $15 \mathrm{~nm}$ of period) and the corresponding onset, but the variational one gives a vanishing binding energy for very small periods. Conversely, the variationalanalytical model of Pereira and Galbraith [14] provides good values only for periods smaller than $10 \mathrm{~nm}$, where the Wannier approximation stands. The variational treatment proposed by Chomette et al. [9], using a rather simple trial function, exhibits the boundary between the two extreme regimes : both in-plane and on-axis extension parameters present a discontinuity at this point. Our heuristic approach allows us to avoid such a snag and gives good effective Rydbergs over the whole range of periods.

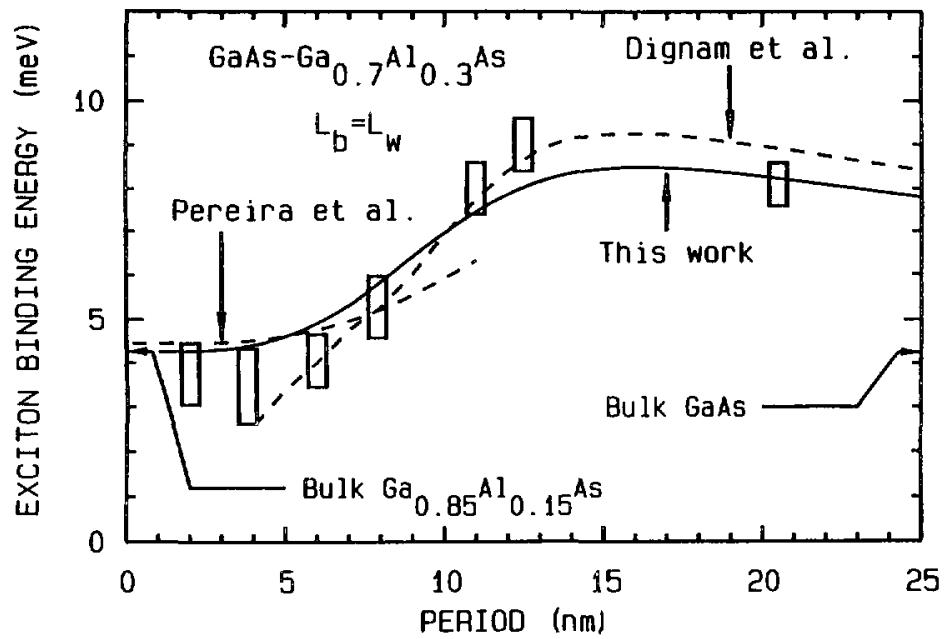

Fig. 4. - Exciton binding energies in symmetrical $\mathrm{GaAs}-\mathrm{Ga}_{07} \mathrm{Al}_{03} \mathrm{As}$ superlatrices, versus the period. Dashed lines show the two extreme situations as calculated by Dignam et al. [13] and Pereira et al. [14]. while a solid line displays the result of using equations (1) and (4). We used here the parameters of reference [13]. Rectangles represent experimental data obtained by Chomette et al. [9] via temperaturedependent photoluminescence excitation spectroscopy. 


\section{Quantum well wires.}

In linear QWW's, electrons and holes are confined along a given plane, while a single direction of free propagation is left. $\alpha$ should thus vary continuously from 3 to 1 . By simple analogy with equation (2), we can propose the following expressions for the dimensionality of the motion of the bound electron-hole pair :

$$
\begin{aligned}
& \alpha=3-\mathrm{e}^{-L_{1}^{*} / 2 a_{0}}-\mathrm{e}^{-L_{1}^{*} / 2 a_{01}} \\
& \alpha=3-2 \cdot \mathrm{e}^{-D^{*} / 2 a_{0}}
\end{aligned}
$$

Equation (5a) stands for a rectangular QWW of lateral effective widths $L_{1}^{*}$ and $L_{1}^{*}$, while equation (5b) describes the case of a cylindrical QWW of effective diameter $D^{*}$. The definition for effective sizes is the same as above. For instance, in a finite-potential $\left(V_{0}\right)$ cylindrical QWW with radius $\rho_{0}$, the envelope function for a carrier is given by [32] :

$$
\Psi(\rho, \theta, z)= \begin{cases}C_{1} J_{\mathrm{m}}\left(k_{\mathrm{w}} \rho\right) \mathrm{e}^{i m \theta} \mathrm{e}^{i h_{-}=}, & \text {for } \rho<\rho_{0} \\ C_{2} K_{\mathrm{m}}\left(k_{\mathrm{b}} \rho\right) \mathrm{e}^{i m \theta} \mathrm{e}^{i k_{z}=}, & \text { for } \rho \geqslant \rho_{0} .\end{cases}
$$

$J_{\mathrm{m}}$ and $K_{\mathrm{m}}$ are the Bessel function and modified Bessel function (Hankel function). $m$ is a rotation quantum number, $k_{\mathrm{w}}$ and $k_{\mathrm{b}}$ are the magnitudes of the in-plane wave vectors inside and outside the wire, respectively and $k_{z}$ is the quantum number for the free propagation along the axis of the QWW ( $z$ axis). $D^{*}$ could thus be defined as :

$$
D^{*}=2 \cdot \rho_{0}+2 / k_{\mathrm{b}} \text {. }
$$

Figure 5 shows a comparison between binding energies calculated versus the QWW radius. The solid curve was obtained by using equations ( $5 b)$ and (7), while the dashed curve was taken from reference [10]. A very good agreement is found between both results.

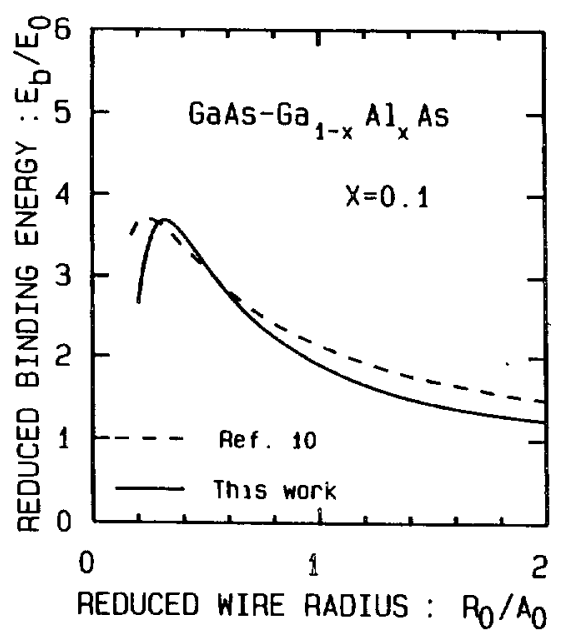

Fig. 5. - Heavy-hole exciton binding energy (in units of the 3D Rydberg) in GaAs- $\mathrm{Ga}_{0,}{ }_{9} \mathrm{Al}_{0}, \mathrm{As}$ quantum well wires, versus the wire radius (in units of the $3 \mathrm{D}$ Bohr radius). The dashed line shows the result obtained by Brown and Spector [10], by a variational calculation, while the solid curve corresponds to equations (1), (5b) and (7). 


\section{Conclusion.}

We have presented a heuristic description of the properties of excitons confined in QW's, SL's and QWW's, based on a frictional-dimensional interaction space. Simplicity and flexibility are the principal qualities of this method. A very good agreement is obtained with the results of variational theories and with experimental findings. A better accuracy can even be obtained from microscopic considerations of realistic spatial extensions of the electron-hole relative motions in the quantum-confined system and the three-dimensional exciton. This is the purpose of a forthcoming paper.

\section{Acknowledgements.}

The authors are grateful to Dr. Xing-Fei He for interesting discussions on the limits of the model.

\section{References}

[1] Bastard G., Mendez E. E., Chang L. L. and Esakı L., Phys Rev. B 26 (1982) 1974.

[2] Miller D. A. B., Chemla D. S., Damen T. C., Gossard A. C., Wiegmann W., Wood T. and Burrus C. A., Phys. Rev. B 32 (1985) 1043.

[3] Greene R. L., Bajaj K. K. and Phelps D. E., Phys. Rev. B 29 (1984) 1807.

[4] Priester C., Allan G. and Lannoo M., Phys Rev. B 30 (1984) 7302.

[5] Sanders G. D. and Chang Y. C., Phys. Rev. B 32 (1985) 5517.

[6] Broido D. A. and Sham L. J., Phy's. Rev. B 34 (1986) 3917.

[7] Ekenberg U. and Altarelli M., Phys. Rev. B 35 (1987) 7585.

[8] Zhu B. and Huang K., Phys. Rev. B 36 (1987) 8102.

[9] Chomette A., Lambert B., Deveaud B., Clérot F., Régrény A. and Bastard G., Europhys. Lett. 4 (1987) 461.

[10] Brown J. W. and Spector H. N., Phys. Rev. B 35 (1987) 3009.

[11] Bauer G. E. W. and Ando T., Phys. Rel. B 38 (1988) 6015.

[12] Wu J. W., Phys. Rev'. B 39 (1989) 12944.

[13] Dignam M. M. and Sipe J. E., Phys. Rev. B 41 (1990) 2865.

[14] Pereira M. F. Jr., Galbraith I., Koch S. W. and Duggan G., Phys. Rev. B 42 (1990) 7084.

[15] Tran Thoai D. B., Zimmermann R., Grundmann M. and Bimberg D., Phys. Rev. B 42 (1990) 5906.

[16] Andreani L. C. and Pasquarello A., Phys. Rev B 42 (1990) 8928.

[17] Leavitt R. P. and Little J. W., Phys. Rev. B 42 (1990) 11774.

[18] Campi D. and Villavecchia C., IEEE J. Quantum Electron. 28 (1992) 1765.

[19] Stillinger F. H., J. Math. Phys. 18 (1977) 1224.

[20] He X. F., Solid State Commun. 61 (1987) 53.

[21] He X. F., Solid State Commun. 75 (1990) 111.

[22] He X. F., Phys. Rer. B 42 (1990) 11751.

[23] He X. F., Phys. Rev. B 43 (1991) 2063.

[24] Mathieu H., Lefebvre P. and Christol P., J. Appl. Phys. 72 (1992) 300.

[25] Mathieu H., Lefebvre P. and Christol P., Phys. Rev. B 46 (1992) 4092.

[26] Lefebvre P.. Christol P. and Mathieu H., Phvs. Rev. B 46 (1992) 13603.

[27] Miller R. C., Kleinmann D. A., Tsang W. T. and Gossard A. C., Phys. Rev B 24 (1981) 1134.

[28] Dawson P., Moore K. J., Duggan G.. Ralph H. I. and Foxon C. T. B.. Phys. Rev' B 34 (1986) 6007.

[29] Molenkamp L. W., Bauer G. E. W., Eppenga R. and Foxon C. T., Phys. Rev $B 38$ (1988) 6147.

[30] Petrou A.. Waytena G., Liu X., Ralston J. and Wicks G., Phys. Rev'. B 34 (1986) 7436.

[31] Reynolds D. C., Bajaj K. K., Leak C., Peters G., Theis W., Yu P. W., Alavi K., Colvard C. and Shidlovsky I., Phys. Rev B 37 (1988) 3117.

[32] Sercel P. C. and Vahala K. J., Phvs, Rev B 42 (1990) 3690. 